\title{
Spin-dependent transport in magnetic sandwiches in the effective-mass approximation
}

\author{
V.F. Los ${ }^{1}$, V.N. Saltanov ${ }^{2}$ \\ ${ }^{1}$ Institute for Magnetism, NAS of Ukraine, 36-B, Academician Vernadsky Blvd, 03142 Kyiv, Ukraine \\ ${ }^{2}$ Taras Shevchenko Kyiv National University, 64, Volodymyrska Str., 01003 Kyiv, Ukraine
}

\begin{abstract}
A theory describing a spin-dependent transport of electrons through a thin metallic (or insulator) nonmagnetic layer sandwiched between two ferromagnets is developed in the ballistic regime and current-perpendicular-to-plane (CPP) geometry. The theory is based on the Landauer formalism and the transmission amplitude for the electron Bloch waves with an arbitrary dispersion law travelling from one ferromagnet to another through a nonmagnetic spacer (metallic or insulator). The semiclassical (nonoscillating) part of the magnetoresistance ratio for a metallic spacer is considered in the effective-mass approximation for the sandwich band structure. The parameters defining the value of the giant magnetoresistance (GMR) effect are obtained. It is shown that the electron specular scattering on the interfaces may be the cause for the CPP GMR effect. The influence of the electronic structure on the CPP GMR effect has been studied numerically in the effective-mass approximation.
\end{abstract}

Keywords: giant magnetoresistance effect, ballistic regime, effective-mass approximation.

Manuscript received 14.06.06; accepted for publication 23.10.06.

\section{Introduction}

Transport phenomena in the artificial structures consisting of alternating magnetic and nonmagnetic layers, each of a few atomic layers thick, have been attracting a great deal of interest especially since the discovery of the giant magnetoresistance (GMR) effect in metallic magnetic multilayers [1] and sandwiches of two magnetic layers separated by a nonmagnetic spacer [2]. These structures exhibit GMR under certain conditions. One of them is a possibility to switch between antiparallel (AP) and parallel (P) alignments of magnetization vectors of ferromagnetic layers in a multilayer or sandwich. It can be achieved by various methods, particularly, using a periodic dependence of a sign of the interlayer exchange interaction between ferromagnetic layers on the nonmagnetic layer thickness [3]. On the other hand, a difference between the $\mathrm{P}$ and AP configurations is important because both an electron scattering by defects in a bulk of magnetic layers or at the interfaces and a potential profile for electrons are spin-dependent, i.e., depend on an orientation of an electron spin relatively to the direction of the magnetic layer magnetization. When the layer thicknesses are less than the spin-diffusion length (a spinflip scattering is absent), the spin-dependent scattering (SDS) by defects and a spin-dependent electronic structure of a multilayer or sandwich give rise to the GMR.

Recently, Schep et al. [4] calculated the GMR for a perfect infinite superlattice using an ab initio bandstructure and found that in the ballistic regime for electrons (no scattering by defects at all) the GMR in the currentperpendicular-to-plane (CPP) geometry can be as large as in the diffusive regime where scattering by defects plays a decisive role in determining the resistance. Thus, an important role of a difference in electronic structure between $\mathrm{P}$ and AP configurations of magnetic multilayers in explaining the GMR has been demonstrated. The quantum scattering from wells and a quantum interference effect play an important role in an explanation of the GMR in the case of ballistic regime.

There have been several works treating the GMR in the ballistic regime. They are based mainly on an exact evaluation of the Kubo formula. Mathon et al. [5] have calculated the ballistic CPP GMR of a $\mathrm{Co} / \mathrm{Cu} / \mathrm{Co}$ trilayer using realistic tight-binding bands fitted to $a b$ initio band structures of $\mathrm{Cu}$ and $\mathrm{Co}$. They found that the CPP GMR without impurity scattering can be as high as $90 \%$ and is due solely to quantum reflections of electrons from perfectly flat $\mathrm{Co} / \mathrm{Cu}$ interfaces. Quantum interference effect leads to oscillations of the CPP GMR with the spacer and ferromagnet thickness [5, 6]. The same approach was used by Mathon [7] for numerical calculation of ballistic transport through $\mathrm{Co} / \mathrm{Cu}$ and $\mathrm{Fe} / \mathrm{Cr}$ multilayers of a finite thickness. General Green's function formalism was developed in [8], which is applicable to studying the CPP conductance in magnetic multilayers and tunneling spin valves with general tightbinding Hamiltonians.

Much less attention has been paid to consideration of the giant magnetoresistance effect beyond the tight- 
binding approximation for an electronic spectrum. It is well known that for electrons which contribute most to the conductivity the tight-binding approach is not the best one. In our previous work [9], we obtained the general transmission amplitude for the electron Bloch waves with an arbitrary dispersion law traveling from one magnetic layer to another through a nonmagnetic spacer with a thickness that is much less that the mean free path (ballistic regime for a spacer). The obtained transmission coefficient allowed for treating a magnetoresistance in metallic multilayers or sandwiches with the thickness of magnetic layers exceeding the electron mean free path [9].

In this paper, we apply the Landauer formalism and the electron transmission amplitude obtained in [9] to studying the spin-dependent transport of two semi-infinite ferromagnets separated by thin nonmagnetic layer in the CPP geometry. The nonmagnetic spacer can be, in general, either metallic or insulator. We have considered the case of a metallic spacer and obtained the general expressions for the semi-classical (nonoscillating) part of the GMR ratio. For analytical and numerical study of the CPP GMR, the electronic structure has been modeled by the parabolic spectrum with the spin- and metal-dependent conduction band profile and the spin- and metaldependent effective masses. The semi-classical part of the GMR ratio considered in the effective-mass approximation for the bandstructure allowed us to find the parameters defining the GMR ratio. Numerical calculations have been performed for the full quantum mechanical GMR ratio in the model of free electrons and in the effective-mass approximation with different masses in different layers. Dependencies of the GMR ratio on the electrons polarization, the spacer potential well depth, the spacer thickness and electron masses have been numerically obtained. It has been shown that the modeling a bandstructure in the effective-mass approximation allows for explaining the large values of the GMR in the ballistic regime. The conditions under which the large GMR effect can be reached are obtained in the effectivemass approximation for the electron spectrum.

\section{Transmission amplitude}

Consider two ferromagnetic conductors separated by a nonmagnetic layer (spacer). We suppose that the thickness of a spacer $d$ is much smaller than the electron mean free path but much larger than the Fermi wavelength which is of the order of a lattice parameter. Then the relevant electronic states can be described by the Bloch waves at the Fermi energy. On the contrary, the thickness of both magnetic layers is sufficiently large in order to guarantee the thermal equilibrium for electrons in magnetic layers and continuous electronic spectrum. The interfaces between magnetic and nonmagnetic substances are considered as the perfect ones and, therefore, the in-plane translational invariance is preserved. Therefore, we assume here that the main contribution to the resistance of the system comes from quantum reflections of electrons from perfectly flat interfaces.
For the system under consideration, the Landauer formalism [10] can be applied for studying the transport phenomena. Since the wave vector $\mathbf{k}_{||}=\left(k^{x}, k^{y}\right)$ parallel to the layers remains a good quantum number, the partial Landauer conductance in some spin channel $G^{\sigma \sigma^{\prime}}$ at zero temperature can be presented as (for the CPP geometry)

$$
G^{\sigma \sigma^{\prime}}=\frac{e^{2}}{h} \sum_{\mathbf{k}_{\|}} T^{\sigma \sigma^{\prime}}\left(\mathbf{k}_{||}\right)
$$

where $T^{\sigma \sigma^{\prime}}\left(\mathbf{k}_{||}\right)$is the transmission coefficient for an electron passing through a nonmagnetic spacer from one magnetic layer, where the projection of an electron spin on the direction of magnetization vector is $\sigma$, to another magnetic layer, where this projection is $\sigma^{\prime}$ (no spin-flip processes are allowed). We might consider a more general situation when $\sigma$ labels also other quantum numbers (e.g., orbital indexes). Here, we suppose that the Fermi level crosses only one band in each layer. The sum in Eq.(1) is over all $\mathbf{k}_{\|}$in the two-dimensional Brillouin zone (the integration over $\mathbf{k}_{\|}$leads to appearance of the spacer cross section $S$, which is supposed to be small enough in the case of a metallic spacer to guarantee a measurable resistance coming mainly from quantum scattering by interfaces between ferromagnets and spacer). In the case of a metallic spacer the summation is performed only over transverse wave vectors corresponding to propagating modes (evanescent states are exponentially localized at the interfaces and do not contribute to the conductance). These wave vectors are defined by a spin-dependent potential profile for spin-up and spin-down electrons in a given configuration of magnetic layers. The transmission coefficient is taken at the energy $E=E_{F}$ that is justifiable for a small applied external voltage.

The conventional magnetoresistance (MR) ratio, defined in terms of the conductances for ferromagnetic (F) and antiferromagnetic (AF) configurations of magnetic layers, is given by

$$
R=\left(G^{\uparrow \uparrow}+G^{\downarrow \downarrow}-2 G^{\uparrow \downarrow}\right) / 2 G^{\uparrow \downarrow}
$$

or

$$
R^{\prime}=\left(G^{\uparrow \uparrow}+G^{\downarrow \downarrow}-2 G^{\uparrow \downarrow}\right) /\left(G^{\uparrow \uparrow}+G^{\downarrow \downarrow}\right)
$$

The arrow up (down) $\uparrow(\downarrow)$ indicates that the electron spin is parallel (antiparallel) to the magnetic layer magnetization. Note, that the summations over transverse wave vectors corresponding to propagating states in Eq. (1) generally are over different areas of $\mathbf{k}_{\mid}$ plane for $G^{\uparrow \uparrow}, G^{\downarrow \downarrow}$ and $G^{\uparrow \downarrow}$. For example, the transmission in the AF case is possible only for the states with the in-plane vectors $\mathbf{k}_{||}$belonging to both sheets of the Fermi surface (corresponding to spin up and spin down) of magnetic layers simultaneously, i.e., when there is an intersection of projections of these sheets on the $\mathbf{k}_{||}$-plane. 
According to [9] the transmission amplitude for an electron moving from one magnetic layer to another one through a nonmagnetic spacer has the form

$$
A\left(k_{1}^{Z}, k_{3}^{Z}\right)=\frac{\alpha_{12}\left(k_{1}^{Z}, k_{2}^{Z}\right) \alpha_{21}\left(k_{2}^{Z}, k_{3}^{Z}\right) \exp \left[i\left(k_{2}^{Z}-k_{3}^{Z}\right) d\right]}{1-\exp \left(2 i k_{2}^{Z} d\right) \alpha_{22}\left(k_{2}^{Z},-k_{2}^{Z}\right) \alpha_{22}\left(-k_{2}^{Z}, k_{2}^{Z}\right)} .
$$

Here $\alpha_{i j}\left(\mathbf{k}_{n}, \mathbf{k}_{n^{\prime}}\right)\left(i, j=1,2 ; n, n^{\prime}=1,2,3\right)$ are the amplitudes of transmission or reflection from $i$-th substance to the $j$-th one. The magnetic substance is numbered by 1 and the nonmagnetic one by 2 . The first variable in the functions $\alpha_{i j}\left(\mathbf{k}_{n}, \mathbf{k}_{n^{\prime}}\right)$ corresponds to the initial incoming electron wave vector and the second one corresponds to the final electron state (transmitted or reflected). All these functions depend on an electron spin. The amplitude [4] describes the situation when the electron Bloch wave with the wavevector $\mathbf{k}_{1}$ travelling from the left magnetic layer through a spacer (where an electron state is characterized by $\mathbf{k}_{2}$ ) experiences multiple scattering by a quantum well/barrier before reaching the second magnetic layer in the state with the wavevector $\mathbf{k}_{3}$. Dependence of amplitudes $\alpha_{i j}$ on $\mathbf{k}_{\| \mid}$is suppressed for brevity in (4) because $\mathbf{k}_{||}$is conserved (zaxis is directed perpendicular to the interfaces). On the other hand, $k_{n}^{Z}$ are the functions of an electron energy $E$ (which is also conserved) and $\mathbf{k}_{\|}$in each layer, $k_{n}^{Z}=\Phi_{n}\left(E, \mathbf{k}_{||}\right)$. Thus, $\alpha_{i j}$ are the functions of $\mathbf{k}_{||}$and $E$.

Note, that $n$, in fact, indicates not only the number of a layer but also the corresponding sheet of the magnetic layer Fermi surface (for a small external voltage we evaluate the transmission coefficient at $E=E_{\mathrm{F}}$ ). The wave vectors $\mathbf{k}_{1}$ and $\mathbf{k}_{3}$ must be equal for $F$ configuration, when the electron states belong to the same sheet of the Fermi surface at both sides of a spacer, whereas these vectors are different for AF configuration.

The electron transmission coefficient is defined as

$T\left(k_{1}^{Z}, k_{3}^{Z}\right)=\frac{V_{3}^{Z}}{V_{1}^{Z}}\left|A\left(k_{1}^{Z}, k_{3}^{Z}\right)\right|^{2}$,

where $V_{3}^{Z}$ and $V_{1}^{Z}$ are the velocity $z$-components for the transmitted and incoming electron states, respectively. The electron velocity is defined in each layer by the corresponding electronic spectrum $E_{n}\left(\mathbf{k}_{n}\right)$ : $\mathbf{V}_{n}=\frac{1}{\hbar} \frac{\partial E_{n}\left(\mathbf{k}_{n}\right)}{\partial \mathbf{k}_{n}}(n=1,2,3)$. Thus, the transmission coefficient essentially depends on the spin-dependent electronic structure of the trilayer under consideration. The transmission coefficient (5) is symmetric over incoming and outgoing electron waves.

In what follows, we will consider the case of metallic spacer using the general formula for the transmission coefficient (5).

\section{Metallic spacer}

In this case, all wave vectors $\mathbf{k}_{n}(n=1,2,3)$ are real. Then, the transmission coefficient (5) can be represented in terms of the transmission coefficients through separate interfaces $\quad T_{1}=\frac{V_{2}^{Z}}{V_{1}^{Z}}\left|\alpha_{12}\left(k_{1}^{Z}, k_{2}^{Z}\right)\right|^{2} \quad$ and $T_{2}=\frac{V_{3}^{Z}}{V_{2}^{Z}}\left|\alpha_{21}\left(k_{2}^{Z}, k_{3}^{Z}\right)\right|^{2}$ as follows $T\left(k_{1}^{Z}, k_{3}^{z}\right)=\frac{T_{1} T_{2}}{\left|1-\exp \left(2 i k_{2}^{Z} d\right) \alpha_{22}\left(k_{2}^{Z},-k_{2}^{Z}\right) \alpha_{22}\left(-k_{2}^{Z}, k_{2}^{Z}\right)\right|^{2}}$.

The formula (6) shows that the transmission coefficient is a periodic function of a spacer thickness $d$ with a period determined by electron wave vector in a nonmagnetic metal (factor $k_{2}^{z} d$ ). Survival of these oscillations of an integration over $\mathbf{k}_{||}$depends on the specific features of the spacer Fermi surface.

Expanding (6) in an infinite series of terms corresponding to an electron transmission through a spacer after any number of subsequent reflections from interfaces within a spacer, we can separate a semiclassical (nonoscillating) part of the transmission coefficient from quantum (oscillating) terms arising due to quantum interference effect. As a result, we have

$T\left(\mathbf{k}_{||}\right)=T_{C}\left(\mathbf{k}_{||}\right)+T_{q}\left(\mathbf{k}_{||}\right)$,

where

$T_{c}\left(\mathbf{k}_{||}\right)=\frac{T_{1} T_{2}}{T_{1}+T_{2}-T_{1} T_{2}}$

and

$T_{q}\left(\mathbf{k}_{||}\right)=T_{1}\left(\mathbf{k}_{||}\right) T_{2}\left(\mathbf{k}_{||}\right) \times$

$\times \sum_{n, m=0}^{\infty} e^{2 i k_{2}^{Z}\left(\mathbf{k}_{||}\right) d(n-m)}\left|C\left(\mathbf{k}_{||}\right)\right|^{n+m} \exp \left[i \varphi\left(\mathbf{k}_{||}\right)(n-m)\right]$.

Here, $C\left(\mathbf{k}_{||}\right)=\alpha_{22}\left(k_{2}^{Z},-k_{2}^{Z}\right) \alpha_{22}\left(-k_{2}^{Z}, k_{2}^{Z}\right)$ is, in general, a complex quantity, which can be presented as $C\left(\mathbf{k}_{||}\right)=\left|C\left(\mathbf{k}_{||}\right)\right| e^{i \varphi\left(\mathbf{k}_{||}\right)}$. We used also that $\left|C\left(\mathbf{k}_{||}\right)\right|^{2}=$ $=\left(1-T_{1}\right)\left(1-T_{2}\right)=R_{1} R_{2}$, where $R_{1}$ and $R_{2}$ are the reflection coefficients from the first and the second interfaces, respectively (in the considered case of a metallic spacer).

Let us consider the semi-classical part of the transmission coefficient more carefully. Restoring indexes of spin projections $\sigma, \sigma^{\prime}$, we can rewrite (8) as

$T_{c}^{\sigma \sigma^{\prime}}\left(\mathbf{k}_{\|}\right) \frac{T_{1}^{\sigma} T_{2}^{\sigma^{\prime}}}{T_{1}^{\sigma}+T_{2}^{\sigma^{\prime}}-T_{1}^{\sigma} T_{2}^{\sigma^{\prime}}}$, 
where $T_{i}^{\sigma}(i=1,2)$ is the transmission coefficient for an electron with fixed spin projection $\sigma$ (relatively to a magnetization vector) moving through a single interface between magnetic and nonmagnetic layers. It is easy to see that $T_{1}^{\uparrow}=T_{2}^{\uparrow}=T^{\uparrow}$ and $T_{1}^{\downarrow}=T_{2}^{\downarrow}=T^{\downarrow}$, i.e., the transmission coefficient through a single interface is the same for an electron moving from one magnetic layer (1) to nonmagnetic spacer (2) and for an electron moving in the opposite direction, if the electron spin projection on the magnetization direction in the magnetic layer is kept fixed. Using this symmetry, one can easily show that

$T_{c}^{\uparrow \downarrow}=2 \frac{T_{C}^{\uparrow \uparrow} T_{C}^{\downarrow \downarrow}}{T_{C}^{\uparrow \uparrow}+T_{C}^{\downarrow \downarrow}}$,

where

$T_{C}^{\uparrow \uparrow}=\frac{T^{\uparrow}}{2-T^{\uparrow}}, T_{c}^{\downarrow \downarrow}=\frac{T^{\downarrow}}{2-T^{\downarrow}}$.

Using the relation (11), we can present the GMR ratio (2) for the semi-classical transmission coefficient as

$$
R_{C}=\frac{\sum_{\mathbf{k}_{||}=0}^{\min \left(\mathbf{k}_{||}^{\downarrow}, \mathbf{k}_{||}^{s}\right)} \frac{\left(T_{C}^{\uparrow \uparrow}-T_{C}^{\downarrow \downarrow}\right)^{2}}{T_{C}^{\uparrow \uparrow}+T_{C}^{\downarrow \downarrow}}+\sum_{\mathbf{k}_{\|}=\min \left(\mathbf{k}_{||}^{\downarrow}, \mathbf{k}_{||}^{s}\right)}^{\min \left(\mathbf{k}_{||}^{\uparrow}, \mathbf{k}_{||}^{s}\right)} T_{C}^{\uparrow \uparrow}}{\sum_{\mathbf{k}_{\|}=0}^{\min \left(\mathbf{k}_{\|}^{\downarrow}, \mathbf{k}_{||}^{s}\right)} 4 \frac{T_{C}^{\uparrow \uparrow} T_{C}^{\downarrow \downarrow}}{T_{C}^{\uparrow \uparrow}+T_{C}^{\downarrow \downarrow}}} .
$$

Here, $\mathbf{k}_{\|}^{\uparrow}, \mathbf{k}_{\| \mid}^{\uparrow}$ and $\mathbf{k}_{\|}^{s}$ are the maximum twodimensional transverse vectors restricting the area of propagating modes for spin-up, spin-down and spacer electrons, respectively. Eq. (13) is valid only if the twodimensional area of propagating wave vectors for the spin-down electrons does not exceed that for the spin-up electrons $k_{\|}^{\uparrow} \geq k_{\|}^{\downarrow}$. If $k_{\|}^{\downarrow} \geq k_{\|}^{\uparrow}$, the GMR ratio is given by Eq. (13) with the reversed arrows' directions ( $\hookrightarrow \downarrow)$ everywhere including the limits of summations. One can see that the GMR ratio (13) is always positive. The relations (12) indicate that the GMR ratio can be represented in terms of the transmission coefficients $T^{\uparrow(\downarrow)}$ corresponding to electron transfer from a magnetic layer to a nonmagnetic spacer (or vice versa)
For $k_{\|}^{\downarrow} \geq k_{||}^{\uparrow}$ Eq. (13) also holds if the substitution $\uparrow \leftrightarrows \downarrow$ is made.

To get more insight into the problem under consideration, we adopt a simple model of a parabolic free-like electronic spectrum in the effective-mass approximation with effective masses being different, in general, in different layers. Then, the spin-dependent potential steps at the interfaces are caused by the difference in the bottom energy of the conduction bands (here we assume that the exchange splitting of bands in a ferromagnet is less than the Fermi energy $E_{\mathrm{F}}$ and $E_{\mathrm{F}}$ exceeds the potential step of a spacer). In this case, the amplitudes of transmission and reflection $\alpha_{i j}$ take the form $\alpha_{12}\left(k_{1}^{Z}, k_{2}^{Z}\right)=\frac{2 V_{1}^{Z}}{V_{1}^{Z}+V_{2}^{Z}}, \alpha_{21}\left(k_{2}^{Z}, k_{3}^{Z}\right)=\frac{2 V_{2}^{Z}}{V_{2}^{Z}+V_{3}^{Z}}$,
$\alpha_{22}\left(-k_{2}^{Z}, k_{2}^{Z}\right)=\frac{V_{2}^{Z}-V_{1}^{Z}}{V_{1}^{Z}+V_{2}^{Z}}, \alpha_{22}\left(k_{2}^{Z},-k_{2}^{Z}\right)=\frac{V_{2}^{Z}-V_{3}^{Z}}{V_{2}^{Z}+V_{3}^{Z}}$.

Using this result, we get from (6) [9]

$T\left(k_{1}^{Z}, k_{3}^{Z}\right)=$

$=\frac{4 V_{1}^{Z}\left(V_{2}^{Z}\right)^{2} V_{3}^{Z}}{\left[\left(V_{2}^{Z}\right)^{2}-\left(V_{1}^{Z}\right)^{2}\right]\left[\left(V_{2}^{Z}\right)^{2}-\left(V_{3}^{Z}\right)^{2}\right] \sin ^{2}\left(k_{2}^{Z} d\right)+\left(V_{1}^{Z}+V_{3}^{Z}\right)^{2}\left(V_{2}^{Z}\right)^{2}}$

We remind that $V_{1}^{Z} \neq V_{3}^{Z}$ in the case of AF arrangement of magnetic layers, and $V_{2}^{Z}$ corresponds to the electron velocity in a spacer. The transmission coefficient for an electron with a given spin projection on the magnetization direction ( $\uparrow$ or $\downarrow$ ) moving from a magnetic layer to a spacer in this case may be written as $T^{\uparrow}=\frac{4 V^{\uparrow} V^{s}}{\left(V^{\uparrow}+V^{s}\right)^{2}}, T^{\downarrow}=\frac{4 V^{s} V^{\downarrow}}{\left(V^{s}+V^{\downarrow}\right)^{2}}$,

where we introduced the notations

$V^{\uparrow}=V_{1}^{Z}\left(\mathbf{k}_{\|}, E_{\mathrm{F}}\right), V^{\downarrow}=V_{3}^{Z}\left(\mathbf{k}_{\|}, E_{\mathrm{F}}\right)$,

$V^{S}=V_{2}^{Z}\left(\mathbf{k}_{\|}, E_{\mathrm{F}}\right)$.

Substituting (17) into (14), we get

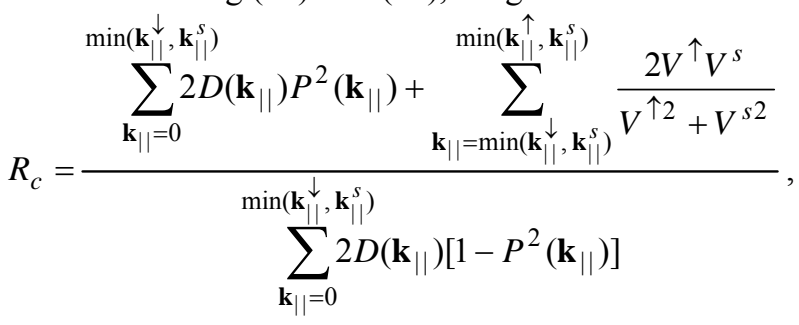

$$
R_{C}=\frac{\sum_{\mathbf{k}_{||}=0}^{\min \left(\mathbf{k}_{\|}^{\downarrow}, \mathbf{k}_{||}^{S}\right)} \frac{2\left(T^{\uparrow}-T^{\downarrow}\right)^{2}}{\left(2-T^{\uparrow}\right)\left(2-T^{\downarrow}\right)\left(T^{\uparrow}+T^{\downarrow}-T^{\uparrow} T^{\downarrow}\right)}+\sum_{\mathbf{k}_{\|}=\min \left(\mathbf{k}_{\|}^{\downarrow}, \mathbf{k}_{||}^{S}\right)}^{\min \left(\mathbf{k}_{\|}^{\uparrow}, \mathbf{k}_{||}^{S}\right)} \frac{T^{\uparrow}}{2-T^{\uparrow}}}{\sum_{\mathbf{k}_{\|}=0}^{\min \left(\mathbf{k}_{\|}^{\downarrow}, \mathbf{k}_{\|}^{S}\right)} \frac{2 T^{\uparrow} T^{\downarrow}}{T^{\uparrow}+T^{\downarrow}-T^{\uparrow} T^{\downarrow}}},\left(k_{\|}^{\uparrow} \geq k_{||}^{\downarrow}\right) .
$$

(C) 2006, V. Lashkaryov Institute of Semiconductor Physics, National Academy of Sciences of Ukraine 
where

$$
P\left(\mathbf{k}_{||}\right)=\left(\frac{V^{\uparrow}-V^{\downarrow}}{V^{\uparrow}+V^{\downarrow}}\right)\left(\frac{V^{s 2}-V^{\uparrow} V^{\downarrow}}{V^{s 2}+V^{\uparrow} V^{\downarrow}}\right)
$$

is the effective spin polarization factor for the ferromagnet-spacer electron transmission, and

$$
D\left(\mathbf{k}_{||}\right)=\frac{V^{s}\left(V^{\uparrow}+V^{\downarrow}\right)\left(V^{s 2}+V^{\uparrow} V^{\downarrow}\right)}{\left(V^{\uparrow 2}+V^{s 2}\right)\left(V^{\downarrow 2}+V^{s 2}\right)} .
$$

Eqs (20) and (21) are the generalization of the corresponding factors, which appeared in the freeelectron theory of the tunneling magnetoresistance effect [11]. When $k_{\|}^{\downarrow} \geq k_{\|}^{\uparrow}$, in Eq.(19) all arrows should be reversed $(\uparrow \hookrightarrow \downarrow)$.

To obtain the total $R(2)$, the quantum part of it, defined by the quantum part of the transmission coefficient (9), should be included in (14) or (19).

\section{Numerical results}

A numerical calculation of the CPP GMR has been performed in the effective-mass approximation when Eqs (15)-(21) are applicable. The influence of the electronic structure on the CPP GMR has been studied in terms of the isotropic effective masses, which may be metal- and spin-dependent, and a constant in each layer spin- and metal-dependent conduction-band profile. Thus, the relevant quantities defining the transport properties of the system take the form

$$
\begin{aligned}
& k^{\uparrow(\downarrow)}=\frac{m^{\uparrow(\downarrow)}}{\hbar} V^{\uparrow(\downarrow)}=\sqrt{\frac{2 m^{\uparrow(\downarrow)}}{\hbar^{2}}} R^{\uparrow(\downarrow)}-\mathbf{k}_{\|}^{2}, \\
& k^{s}=\frac{m^{s}}{\hbar} V^{s}=\sqrt{\frac{2 m^{s}}{\hbar^{2}} R^{s}-\mathbf{k}_{\|}^{2}},
\end{aligned}
$$

where $\quad R^{\uparrow(\downarrow)}=E_{\mathrm{F}}-U^{\uparrow(\downarrow)} \geq 0, \quad R^{s}=E_{\mathrm{F}}-U^{s} \geq 0$. Here $m^{\uparrow(\downarrow)}, m^{s}$ and $U^{\uparrow(\downarrow)}, U^{s}$ are the effective masses and potentials in magnetic layers (for spin-up and spin-down electrons) and in a spacer, respectively. Thus, the maximum values of $\mathbf{k}_{||}$in magnetic layers and a spacer (corresponding to propagating modes), which define the areas of integration in Eq. (19), are

$$
\begin{aligned}
& k^{\uparrow(\downarrow)}=\sqrt{\frac{2 m^{\uparrow(\downarrow)}}{\hbar^{2}} R^{\uparrow(\downarrow)}}, \\
& k^{s}=\sqrt{\frac{2 m^{s}}{\hbar^{2}} R^{s}},
\end{aligned}
$$

They depend in the model under consideration on the potential wells $R^{\uparrow(\downarrow)}, R^{s}$ and the effective masses $m^{\uparrow(\downarrow)}, m^{s}$ in different layers.
Eqs (19)-(21) indicate that there are several factors influencing the MR ratio, such as, e.g., the spin polarization

$$
P_{0}=\frac{V_{0}^{\uparrow}-V_{0}^{\downarrow}}{V_{0}^{\uparrow}+V_{0}^{\downarrow}}
$$

taken at $\mathbf{k}_{\|}=0$, and the overlapping of the spin-up and spin-down Fermi surface leaves projections on the $\mathbf{k}_{||-}$ plane corresponding to propagating states. This overlapping is defined by the minimal value among the wave vectors (23). The second terms in Eqs (13), (14) and (19) are defined by the difference of the $\mathbf{k}_{\mathrm{l}}$-planes contributing to the conductance in the parallel and antiparallel configurations of magnetization directions in magnetic layers. As it is seen from Eqs (22) and (23), the electron velocities and the integration areas in Eq. (18) over the $\mathbf{k}_{||}$-plane depend on the depth of the potential wells $R^{\uparrow(\downarrow)}, R^{s}$ and the electron effective masses in different layers as well. Thus, $R^{\uparrow(\downarrow)}, R^{S}$ and $m^{\uparrow(\downarrow)}$, $m^{s}$ are also the important parameters of the model under consideration.

First, we will consider the dependence of the MR ratio on the electron polarization $P_{0}(24)$. Because $\left|P_{0}\right|$ changes from 0 to 1 , it is reasonable to calculate the dependence on $P_{0}$ of the MR ratio $R^{\prime}$ defined by Eq.(3) where $G^{\sigma \sigma^{\prime}}$ is defined by Eq. (1). Numerical evaluation of the conductances $G^{\sigma \sigma^{\prime}}$ has been performed using Eqs (1) and (16) for the transmission coefficient, which is exact in the effective-mass approximation and takes into account the quantum interference effects. These effects manifest themselves through the dependence of the transmission coefficient (16) on the thickness of a spacer $d$ and the electronic wavelength in a spacer $\sim\left(k_{2}^{z}\right)^{-1}$. The area of integration over $\mathbf{k}_{||}$in each term of Eq. (3) is restricted to the minimal value among the wavevectors (23). For simplicity, the calculation was performed, first, for free electrons, when $m^{\uparrow(\downarrow)}=m^{s}=$ $m$, where $m$ is the free electron mass. The calculated dependence of the MR ratio (3) on $P_{0}$ at different spacer thicknesses and potential wells is shown in Fig. 1.

This dependence exhibits several characteristic features. When the spacer potential well is not very deep (Fig. 1a), the MR ratio at small values of $P_{0}$ differs not much for different spacer thicknesses and increases monotonically and not very fast. At small $P_{0}$ and $R^{s}<<R^{\downarrow}<R^{\uparrow}$, the integration domain for all $G^{\sigma \sigma^{\prime}}$ is defined by $R^{S}$ and remains constant while $R^{\downarrow} \geq R^{S}$. With increasing $P_{0}$ (increasing $R^{\uparrow}-R^{\downarrow}$ and decreasing $R^{\downarrow}$ ) the MR ratio reaches some intermediate maximum at $R^{\downarrow}=R^{S}$. At $R^{\downarrow}<R^{s}$ the area of integration over $\mathbf{k}_{\mid}$ for $G^{\downarrow \downarrow}$ and $G^{\uparrow \uparrow}$ is defined by $R^{\downarrow}$ and diminishes with increasing $P_{0}$. Then, the oscillations of the MR ratio 
occurs, the amplitudes of which depend on the spacer thickness. At some spacer thicknesses, the amplitude of the oscillation is so large that the MR ratio becomes negative. These large oscillations are due to the quantum interference effect. For sufficiently large $P_{0}$, the MR ratio $R^{\prime}$ reaches a significant value and tends to the maximum value $R^{\prime}=1$.

When a spacer potential well is deep enough (Fig. 1b), the MR ratio varies smoothly (oscillations are small) and does not depend significantly on the spacer thickness.

In what follows, the MR ratio defined by Eq. (2) will be used for numerical calculation as well as Eqs (1) and (16). Dependence of the MR ratio $R$ on the depth of a potential well $R^{S}$ calculated for free electrons is shown in Fig.2. At $R^{s}<R^{\downarrow}$ the MR ratio is small and changes with $R^{S}$ slowly. In the range $R^{\downarrow} \leq R^{S} \leq R^{\uparrow}$ it increases sharply by $10-30$ times and reaches a maximum at $R^{S}=R^{\uparrow}$. This maximum value of the MR ratio may be estimated at the considered small value of $P_{0}$ as

$R_{\max } \approx\left(R^{\uparrow}-R^{\downarrow}\right) /\left(2 R^{\downarrow}\right),\left(R^{s}=R^{\uparrow}\right)$

where $R^{\uparrow}>R^{\downarrow}$ for the model of free electrons. Then, at $R^{S}>R^{\uparrow}$ the function $R\left(R^{S}\right)$ fluctuates around some average value which is slightly smaller than $R_{\max }$.
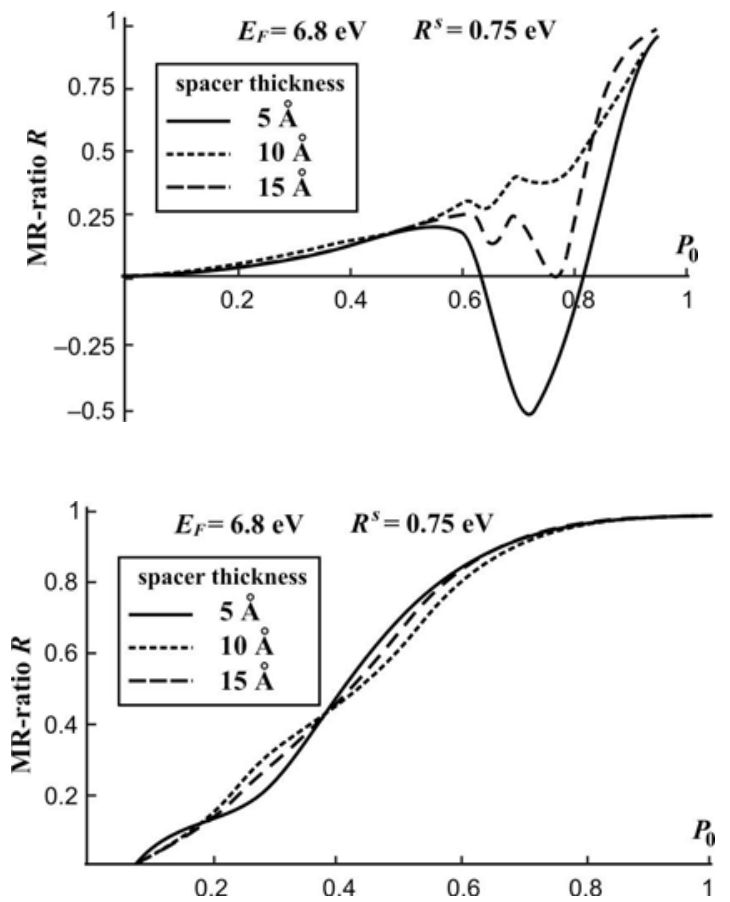

Fig. 1. MR-ratio dependence on the polarization $P_{0}$ for not very deep (a) and deep (b) potential well.
Such a behavior may be understood in terms of semi-classical Eq. (19) for the MR ratio. At $R^{S}<R^{\downarrow}<R^{\uparrow}$ the second term in Eq. (19) is absent and the MR ratio is small when $P_{0}<<1$. When $R^{S}$ reaches $R^{\downarrow}$, then the second term in Eq. (18) emerges and begins contributing to $R_{C}$. Its contribution rises with $R^{S}$ because the integration domain in the second term of the numerator is equal to $k_{\|}^{s}-k_{\|}^{\downarrow}$ and reaches the maximum value $k_{\|}^{\uparrow}-k_{\|}^{\downarrow}$ when $R^{S}=R^{\uparrow}$. The integrand of this term $T^{\uparrow \uparrow}$ also increases with $R^{S}$ and reaches its maximum value $T^{\uparrow \uparrow}=1$ when the majority band (spin-up) and the electronic band in a spacer become equivalent (at $R^{S}=R^{\uparrow}$ ). Then, the integration over $k_{\|}^{2}$ in the second term of the numerator of Eq. (18) at $R^{S}=R^{\uparrow}$ results in $2 \pi m\left(R^{\uparrow}-R^{\downarrow}\right) / \hbar^{2}$. The denominator of Eq. (19) at $P\left(\mathbf{k}_{||}\right)<<1$ and $R^{S}=R^{\uparrow}$ gives $4 \pi m R^{\downarrow} / \hbar^{2}\left(D\left(\mathbf{k}_{||}\right) \approx 1\right.$ when the electron polarization is small, $V^{\uparrow} \approx V^{\downarrow}$. It easy to see from Eqs (19)-(21) that, when the polarization $P$ is small and $R^{S} \approx R^{\uparrow}$, the contribution of the second term to Eq. (19) becomes dominant. Further $R^{S}$ increasing does not change the integration domain in the second term of Eq. (19) but the transmission coefficient $T^{\uparrow \uparrow}$ and the factor $D$ in Eq. (19) become smaller, i.e., the MR ratio $\mathrm{R}$ diminishes on average.

Fig. 2 indicates that the maximum value of the MR ratio $R$ as a function of a potential well depth $R^{S}$ depends not only on the electron polarization $\left(R^{\uparrow}-R^{\downarrow}\right)$ but also on the absolute values of $R^{\uparrow}$ and $R^{\downarrow}$. The bigger the difference $R^{\uparrow}-R^{\downarrow}$ the larger the MR ratio. At fixed $R^{\uparrow}-R^{\downarrow}$, the smaller the absolute values of $R^{\uparrow}$ and $R^{\downarrow}$ the bigger MR ratio $R$. Such a behavior agrees with the estimate (25) for the maximum value of $R$.

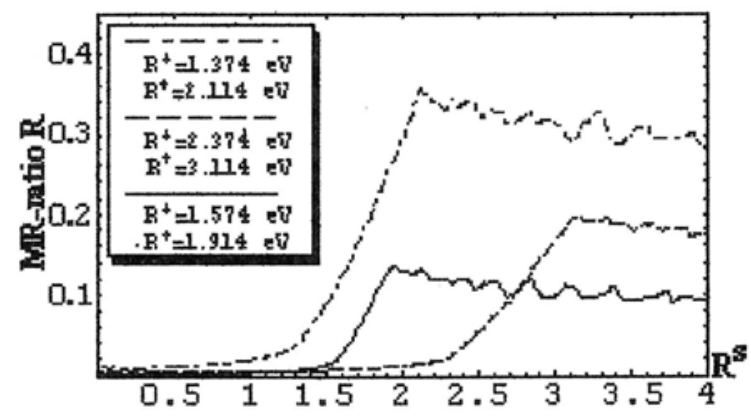

Fig. 2. MR-ratio dependence on the potential well depth $R_{S}$ for different values of $R_{\downarrow}$ and $R^{\uparrow}$. 


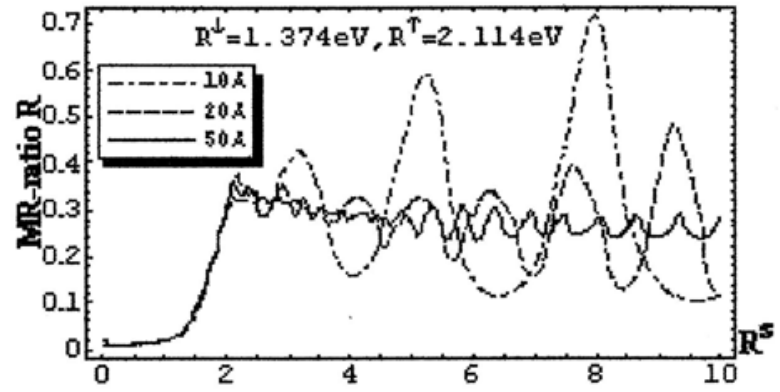

Fig. 3. MR-ratio dependence on the potential well depth $R^{S}$ for different spacer thicknesses $d$.

Oscillations of the MR ratio seen in Fig. 2 are caused by the quantum interference effect and can not be described by the classical Eq. (19). It is evident from Eq. (16) for the transmission coefficient, that the spacer thickness $d$ should influence the period and amplitude of these oscillations. The dependence of $R\left(R^{S}\right)$ at different spacer thicknesses is shown in Fig. 3. The MR ratio $R\left(R^{S}\right)$ oscillates around some average value of $R$ which does not depend on $d$ and is approximately equal to $R_{\max }$ (25). The bigger $d$ the smaller the period and amplitude of oscillations.

In the free-electron approximation, there is no other way to increase the MR ratio but raising the electron polarization $P_{0}$ which for free electrons is

$$
P_{0}=\left(\sqrt{R^{\uparrow}}-\sqrt{R^{\downarrow}}\right) /\left(\sqrt{R^{\uparrow}}+\sqrt{R^{\downarrow}}\right) .
$$

However, the electron polarization in ferromagnets is normally not very high, $P_{0}<<1$, and defined by an internal effective magnetic field. Thus, the maximum value of the MR ratio (25) in this model can not be made large enough with an exception of the case, when the magnetic layers are made of semi-metals where $P_{0}$ is close to unity [12].

In the effective-mass approximation, however, there are more parameters to adjust a theory to an experiment and to show a way to finding the new materials with a large GMR effect. We have seen that the MR ratio sharply increases at the expense of the second term in Eq. (19) which is proportional (roughly) to the integration domain of the numerator of this term (see Eq. (25)). In the effective-mass approximation, the maximum value of this integration area is $2\left|m^{\uparrow} R^{\uparrow}-m^{\downarrow} R^{\downarrow}\right| / \hbar^{2}$ and is achieved when

$m^{s} R^{s}=\max \left(m^{\uparrow} R^{\uparrow}, m^{\downarrow} R^{\downarrow}\right)$.

The latter condition follows from the fact that in the effective-mass approximation the maximum value $k_{\|}^{\uparrow}$ (22) may become smaller than $k_{\|}^{\downarrow}$ due to the effective masses $m^{\uparrow}$ and $m^{\downarrow}$ difference (although
$R^{\uparrow}>R^{\downarrow}$ by definition). If $k_{\|}^{\downarrow} \geq k_{\|}^{\uparrow}$, then in Eq. (19) all the arrows should be reversed. Thus, in the effectivemass approximation, one might expect that at $m^{s} R^{S}=\max \left(m^{\uparrow} R^{\uparrow}, m^{\downarrow} R^{\downarrow}\right)$ the maximum value of the MR ratio would be

$$
R_{\text {max }} \approx\left|m^{\uparrow} R^{\uparrow}-m^{\downarrow} R^{\downarrow}\right| /\left[2 \min \left(m^{\uparrow} R^{\uparrow}, m^{\downarrow} R^{\downarrow}\right)\right] .
$$

We see that in this approximation there is a possibility to increase the MR ratio by assigning the different effective electron masses to different layers. Assigning the different effective masses to different layers (the spin-up and spin-down Fermi surface leaves in a magnetic material) is a reasonable thing to do when trying to account for a rather complicated electronic structure of multilayers and sandwiches exhibiting the GMR effect. For numerical calculation, we have considered the case when the effective masses of electrons in a nonmagnetic spacer and for the majority spin (spin-up) in a magnetic layer are equal to a free electron mass, $m^{\uparrow}=m^{s}=m$. This is a good approximation, e.g., for the band structure of $\mathrm{Co}, \mathrm{Ni}$ and $\mathrm{Cu}$ around the Fermi energy. On the other hand, a free electron description for the minority spin is not generally a good one. Thus, we calculated the dependence of the MR ratio on the spin-down effective mass $m^{\downarrow}$, which is shown in Figs 4 and 5. The values of $R^{\uparrow}$ and $R^{\downarrow}$ are selected as the one-site energies in Ni for majority and minority spin, respectively. The thickness of the spacer is selected to be big enough, $d=50 \AA$, in order to avoid significant fluctuations, which may shadow the main trend of the MR ratio change.

Fig. 4 shows the dependence of the MR ratio (2) on the spacer potential well depth, $R\left(R^{S}\right)$, for $m^{\downarrow}=1$ and $m^{\downarrow}=8$ (in the free electron mass units). The first line (I) is identical to that for the free electron case (solid line in Fig. 2) with the maximum value approximated by Eq. (25). The maximum of the second line (II) $\left(m^{\downarrow}=8\right)$ is reached when $m^{s} R^{s}=m^{\downarrow} R^{\downarrow}$ (here $m^{\downarrow} R^{\downarrow}>m^{\uparrow} R^{\uparrow}$ )

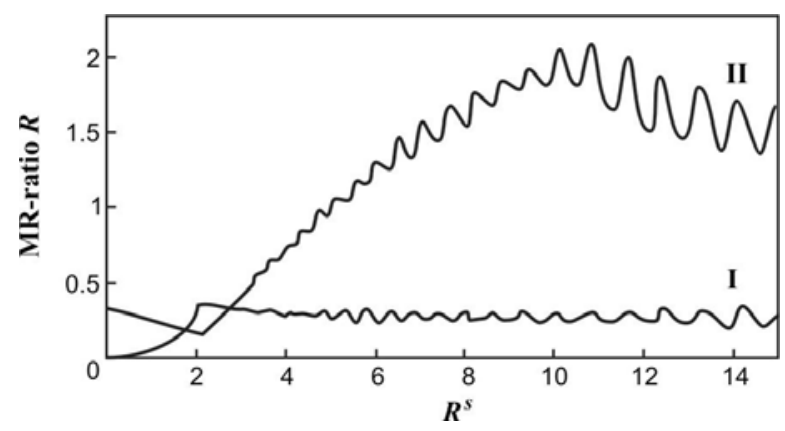

Fig. 4. MR-ratio dependence on the value $m^{s} R^{s}$. I - for the value of $m^{\downarrow}=1$. II - for the value of $m^{\downarrow}=8$. 
and may be approximated by (28), which for the selected parameters is $R_{\max } \approx 2.1$. The origin of this maximum may be explained in the same way as it was done for the free-electron approximation (line I in Fig. 4). When $m^{s} R^{s} \leq m^{\uparrow} R^{\uparrow}$, the second term in Eq. (19) is absent $\left(k_{\|}^{\downarrow} \leq k_{\|}^{s}<k_{\|}^{\uparrow}\right)$, and the MR ratio changes slowly. At $m^{s} R^{s}>m^{\uparrow} R^{\uparrow}$ the second term in Eq. (19) emerges $\left(k_{\|}^{\uparrow}<k_{\|}^{s}<k_{\|}^{\downarrow}\right)$ and increases with $m^{s} R^{s}$ because the area of integration enlarges. The maximum is reached at $m^{s} R^{s}=m^{\downarrow} R^{\downarrow}\left(k_{\|}^{s}=k_{\|}^{\downarrow}\right)$ when the integration domain of the term under consideration becomes maximal and does not increase with the further increasing of $k_{\|}^{s}$.

In order to study the dependence of the MR ratio (2) on $m^{\downarrow}$, we will fix the only free parameter left - the spacer potential well depth $R^{S}$ (we remind that $\left.m^{\uparrow}=m^{s}=m\right)$. Fig. 5 shows the calculated dependence $R\left(m^{\downarrow}\right)$ at the large (line II) and small (line I) value of $R^{S}$, when $R^{S}=7 \mathrm{eV}>R^{\uparrow}>R^{\downarrow}$ and $R^{S}=R^{\uparrow}=$ $=2.114 \mathrm{eV}$, respectively. The line III shows the dependence of the maximum value (28) on $m^{\downarrow}$, $R_{\max }\left(m^{\downarrow}\right)$. All three lines exhibit a minimum at $m^{\uparrow} R^{\uparrow}=$ $=m^{\downarrow} R^{\downarrow}$. It is evident for Eq. (28), and, according to Eq. (18), the increase of $m^{\downarrow} R^{\downarrow}$ up to $m^{\uparrow} R^{\uparrow}$ leads in the considered cases to the decreasing of the second term in Eq. (19), which becomes equal to zero at $k_{\|}^{\uparrow}=k_{\|}^{\downarrow}$. Further increasing of $m^{\downarrow}$ in the case, when $R^{S}>R^{\uparrow}>R^{\downarrow}$ (line II), leads to the non-zero and increasing second term of Eq. (19) (with reversed arrows at $k_{\|}^{\downarrow}>k_{\|}^{\uparrow}$ ) which results in the sufficiently sharp increase of the MR ratio. At $m^{\downarrow} R^{\downarrow}<m^{s} R^{s}$ and up to the value $m^{\downarrow} R^{\downarrow}=m^{s} R^{s}$ the MR ratio increases rapidly (more general at $m^{s} R^{s} \geq \max \left(m^{\uparrow} R^{\uparrow}, m^{\downarrow} R^{\downarrow}\right)$ ). It is interesting that the line II practically coincides with $R_{\max }\left(m^{\downarrow}\right.$ ) (line III) in the considered range of $m^{\downarrow}$ (up to $k_{\|}^{\downarrow}=k_{\|}^{s}$. Further increasing of $m^{\downarrow}$ in the case $R^{s}>$ $>R^{\uparrow}>R^{\downarrow}$ (line II) does not result in the enlarging of Eq. (19) second term integration area, and $R\left(m^{\downarrow}\right)$ increases at $m^{\downarrow} R^{\downarrow}>m^{s} R^{s}$ slowly.

In the case when $R^{S}=R^{\uparrow}$ (line I), the MR ratio drops with $m^{\downarrow}$ practically in accordance with the line III $\left(R_{\max }\left(m^{\downarrow}\right)\right)$ till $\quad m^{\downarrow} R^{\downarrow}=m^{\uparrow} R^{\uparrow}=m^{s} R^{s}$ and then increases very slowly, remaining small in the shown in

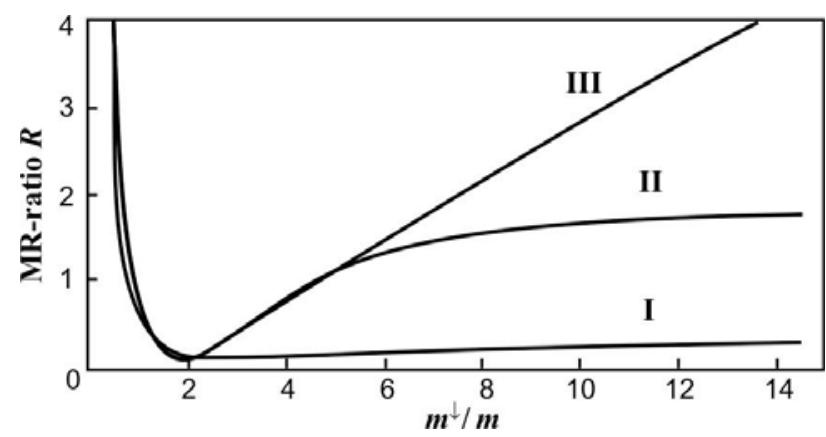

Fig. 5. GMR-ratio dependence on $m^{\downarrow}$ : I - for the small value of $R^{S}\left(R^{S}=R^{\uparrow}=2.114 \mathrm{eV}\right)$, II - for the large value of $R^{S}\left(R^{S}=\right.$ $=7.0 \mathrm{eV}$ ). III - dependence of the maximum value (Eq. 27) on $m^{\downarrow}$.

Fig. 5 range of $m^{\downarrow}$ change $\left(m^{\downarrow} R^{\downarrow}>m^{\uparrow} R^{\uparrow}\right)$. Again, such a behavior of the MR ratio in this case corresponds to the contribution of the second term of Eq. (19), which drops to zero with the increasing of $m^{\downarrow} R^{\downarrow}$ up to $m^{\uparrow} R^{\uparrow}$ and remains zero at $k_{\|}^{\downarrow} \geq k_{\|}^{\uparrow}=k_{\|}^{s}$.

Thus, the effective-mass approximation, the simplest one allowing to account for a rather complicated magnetic sandwich band structure, makes it possible to explain the large values of the MR ratio observed experimentally in sandwiches exhibiting the GMR effect. These values can be reached at the large enough spin-down electron mass $m^{\downarrow}$, which reflects the well known fact that the spin-down band at the Fermi energy is a $d$-like band and the minority-electron current is carried by heavy $d$-electrons.

\section{Conclusions}

We have considered the CPP GMR in magnetic sandwiches with the semi-infinite magnetic layers taking into account the specific features of the band structure in the effective-mass approximation. The classical part of the MR ratio (19) has been obtained in this approximation. It allows the interpretation of the numerical results in terms of the parameters entering Eq. (19). Thus, the condition under which the maximum MR ratio can be achieved in this model is given by Eq. (27). The maximum MR ratio value has been estimated by Eq. (28). In the model of free electrons, these results become equal to Eq. (25). The main contribution to the MR ratio is defined by the difference of the $\mathbf{k}_{\mid}$-planes restricting propagating electron modes in the $\mathrm{F}$ and $\mathrm{AF}$ configurations of magnetic layers. It follows from Eq. (28) that the difference of the electron masses in different layers may be crucial for achieving the sizeable GMR effect in magnetic sandwiches because the electron polarization (26) can not be usually made big enough. 
Numerical calculation performed using the exact quantum formulas (1), (2) and (16) confirmed that to achieve a sizeable GMR effect a magnetic sandwich should be made up of magnetic layers with a big enough $R_{\max }$ (28) divided by a nonmagnetic spacer with $m^{s} R^{s} \gtrsim \max \left(m^{\uparrow} R^{\uparrow}, m^{\downarrow} R^{\downarrow}\right)$. Particularly, it can be realized in magnetic materials with the heavy spin-down electrons.

\section{Acknowledgement}

The research described in this publication was made possible in part by Award \#UP2-2117 of the U.S. Civilian Research \& Development Foundation for the Independent States of the Former Soviet Union (CRDF).

\section{References}

1. M.N. Baibich, J.M. Broto, A. Fert, F. Nguen Van Dau, F. Petroff, P. Etienne, G. Creuzet, A. Friederich, and J. Chazelas // Phys. Rev. Lett. 61, 2472 (1988).
2. G. Binasch, P. Grünberg, F. Saurenbach, and W. Zinn // Phys. Rev. B 39, 4828 (1989).

3. S.S. P. Parkin, N. More, and K.P. Roche // Phys. Rev. Lett. 64, 2304 (1990).

4. K.M. Schep, P. Kelly, and G. E. Bauer// Phys. Rev. Lett. 74, 586 (1995).

5. J. Mathon, A. Umerski, and Murielle Villert // Phys. Rev. B 55, 14378 (1997).

6. J. Mathon, Murielle Villert, and H. Itoh // Phys. Rev. B 52, R6983 (1995).

7. J. Mathon // Phys. Rev. B 55, 960 (1997).

8. S. Sanvito, C.J. Lambert, J.H. Jefferson, and A.M. Bratkovsky // Phys. Rev. B 59, 11936 (1999).

9. V.F. Los and A.N. Pogorily // J. Phys. D: Appl. Phys. 33, 1267 (2000).

10. S. Datta, Electronic Transport in Mesoscopic Systems. Cambridge University Press, Cambridge, 1995.

11. J.C. Slonczewski // Phys. Rev. B 39, 6995 (1989).

12. A.M. Bratkovsky // Phys. Rev. B 56, 2344 (1997). 